\title{
Making Medical Visual Information Available on the WEB
}

\author{
Silvio Antonio Carro ${ }^{1}$ \\ Jacob Scharcanski ${ }^{2}$
}

\begin{abstract}
This paper presents a new metadata model to describe and retrieve medical visual information, such images and their diagnoses, using the Web. The classes of this model allow describing medical images of different medical areas, including their properties, components and relationships. This model supports the international classification of diseases and related health problems (i.e. ICD-10) [1]. The MedISeek (Medical Image Seek) prototype presented here proposes a medical image sharing system based on web services, that allows authorized users to describe, store and retrieve medical images and their associated diagnostic information,based on the proposed metadata model. Thus, this paper proposes to include the image description, converted to RDF syntax, into a JPEG image and a persistent structure for relational databases to storage and retrieve this metadata, providing fast indexing and querying. A description of the prototype structure also is provided.
\end{abstract}

\section{Introduction}

Healthcare professionals use more and more the Internet to retrieve knowledge reference sources for decision making and educational purposes. Particularly, the design of Internet browsers and query systems for medical image retrieval have attracted the attention of many researchers [2].

Extensive use of xray imaging, and the advent of the digital computer and new imaging modalities, such as magnetic resonance, digital x-ray, computerized tomo graphy, nuclear medicine and ultrasound, have contributed to create an explosion of diagnostic

\footnotetext{
${ }^{1}$ Faculdade de Informática, UNOESTE, Presidente Prudente/SP, Brasil

\{silvio@apec.unoeste.br\}

${ }^{2}$ Instituto de Informática, UFRGS, Porto Alegre/RS, Brasil

\{jacobs@inf.ufrgs.br\}
} 
imaging techniques over the past 25 years [3]. Yet, in some areas of medicine, images are generated using digital cameras, or even sample digitization through a scanner.

Now, techniques for archiving, describing and communicating such images are at need.

The conventional process of image digitization do not consider the image content (i.e. what the image represents), and do not offer the means to describe it. As important as the form that images are presented is how they are interpreted. Unfortunately, the image visual content by itself is not sufficient for the image interpretation. The addition of complementary textual information, promotes expressiveness on images, and may help their retrieval.

The MedISeek system, using the proposed metadata model and a system manager, helps professionals of the medical field to describe their images, and make them available for being searched and retrieved through the web.

Section 2 of this paper describes the proposed metadata model for medical image description and retrieval; section 3 presents the prototype designed to validate the metadata model, described in the section 2; and, finally, section 4 presents some concluding remarks, and discusses our future work.

\section{Image Descriptive Metadata}

Metadata are usually defined as "data that describes data". The use of metadata can be important for controlling web resources; offering organization, identification, safety, description, retrieval, access control and protection of copyrights.

Images, in particular, are constituted by units of information (pixels) with little expressive power, so metadata may help image description and indexing, which makes easier the image retrieval task. Metadata is an important resource when associated with images for several reasons, such as: they supply detailed information about images and image files; allow the indexing, identification, classification (according to a pre-determined schema) to describe the image contents.

Several metadata patterns exist, each one designed for specific applications, for example: Dublin Core (DC) [4] for resources on the web; Machine Readable Catalogue (MARC) [5] for bibliographical cataloguing; Spatial Archive and Interchange Format (SAIF) [6] for spatial and spatial/temporal data; Text Encode Initiative (TEI) [7] for textual representation in electronic format and Summary Object Interchange Format (SOIF) [8] for indexation and retrieval resources on the web. Currently, researchers having been working on ways to provide integration and interoperability of existent metadata patterns.

To reach interoperability between metadata patterns, different architectures have been proposed to support metadata coding by using common semantic conventions, structures and syntax. Among these metadata architectures we can mentioned a few, such as: Meta Content 
Framework (MCF) [9], Warwick Framework [10] and Resource Description Framework (RDF) [11].

Relational databases are an alternative to store and to manipulate these metadata architectures because they provides persistency, and ways to resolve queries about the images. In this work, we propose a solution that is a modification of the metadata model proposed by Zanette [12].

The patterns studied by Zanette are not appropriate for the efficient description of medical images, because they do not supply enough elements to express many of the important characteristics of that resource type. The next section presents the metadata model we propose to describe and retrieval medical images on the web

\subsection{The MedISeek metadata model}

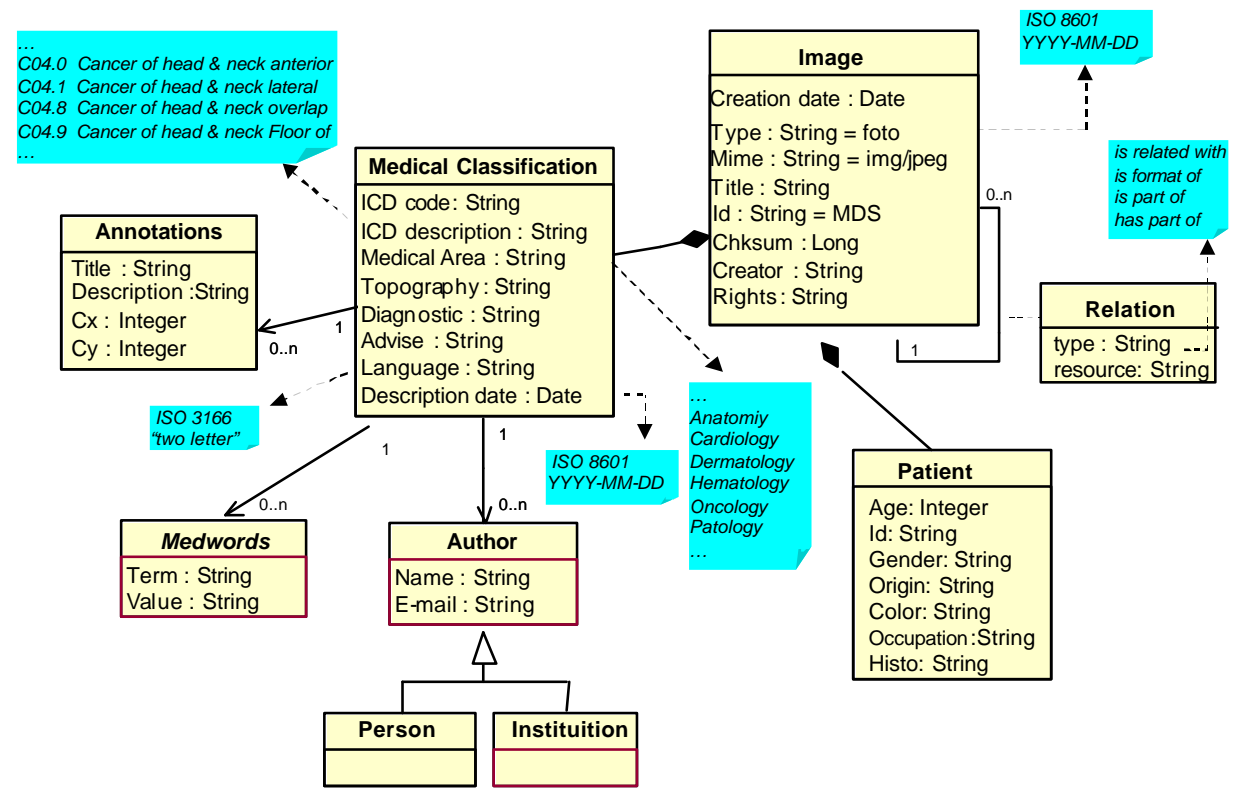

Figure 1. Model class diagram

The proposed model is represented in UML (Unified Modeling Language) in figure 1. This conceptual schema has the purpose of describing medical images of several areas. 
The model is based on five classes: Patient, Medical Classification, Image, Annotations and Medwords. The classes are composed by metadata elements that describe the technical information, the content and the image relationships.

Next, the descriptions of each classes defined in the model are detailed.

\section{Image Class}

The image class represents a group of generic features considered as technical information. The properties of the image class and their descriptions can be visualized in the Table 1 .

Table 1. Image class properties

\begin{tabular}{|c|c|c|}
\hline Element & Description & Value/Format \\
\hline Creation date & $\begin{array}{l}\text { Date associated with the creation or } \\
\text { availability of the resource. Recommended } \\
\text { the ISO } 8601 \text { (W3CDTF) format. }\end{array}$ & $\begin{array}{l}\text { ISO } 8601 \\
\text { (yyyy-mm-dd) }\end{array}$ \\
\hline Type & Original image type & $\begin{array}{l}\text { Photography, picture } \\
\text { or graphical schema }\end{array}$ \\
\hline Mime & MIME type & image/jpeg \\
\hline Id & Value to validate de metadata model. & "MDS" \\
\hline Title & Image title & Free text \\
\hline Chksum & $\begin{array}{l}\text { Numeric value to warrants autenticity into } \\
\text { image and description. }\end{array}$ & Numeric value \\
\hline Creator & $\begin{array}{l}\text { An entity primarily responsible for } \\
\text { making the digital image. }\end{array}$ & $\begin{array}{l}\text { Person, organization, } \\
\text { etc. }\end{array}$ \\
\hline Rights & $\begin{array}{l}\text { Rights element will contain a rights } \\
\text { management statement for the image, or } \\
\text { reference a service providing such } \\
\text { information }\end{array}$ & $\begin{array}{l}\text { URL, bibliographic } \\
\text { reference, author, } \\
\text { organization, etc. }\end{array}$ \\
\hline
\end{tabular}

\section{Patient Class}

The patient class defines attributes that represent the represented person. Table 2 presents the properties of the patient class.

Table 2. Patient class properties

\begin{tabular}{lll}
\hline \multicolumn{1}{r}{ Elemento } & \multicolumn{1}{c}{ Descrição } & \multicolumn{1}{c}{ Valores/Formato } \\
\hline Age & Patient age & Age in years \\
ID & Patient identification. & String
\end{tabular}




\begin{tabular}{|c|c|c|}
\hline Gender & Patient gender. & $\begin{array}{l}\text { Male, female or not } \\
\text { identificated. }\end{array}$ \\
\hline Origin & Origin region & Format: \\
\hline Color & Human skin color or race & $\begin{array}{l}\text { Country|State } \mid \text { City } \\
\text { White, black, yellow, } \\
\text { etc. }\end{array}$ \\
\hline Occupation & Main occupation & Free text \\
\hline Histo & Patient clinical history. & Free text \\
\hline
\end{tabular}

\section{Medical Classification Class}

The Medical classification class represents the description of the visual content related to the attributes of the medical area. One of the elements of this class is international classification of diseases and related health problems, tenth revision (ICD-10) [1], which is an ontology proposed by the World Health Organization to classify diseases, lesions and external causes of diseases/lesions.

Properties of the medical classification class are presented in the Table 3.

Table 3. Medical class properties

\begin{tabular}{lll}
\hline \multicolumn{1}{c}{ Elemento } & \multicolumn{1}{c}{ Descrição } & \multicolumn{1}{c}{ Valores/Formato } \\
\hline ICD & ICD code version 10 & ICD-10 elements \\
ICD desc & ICD description & String \\
Medical area & Medical area related into image & Pathology, oncology, \\
& & radiology, urology, \\
& & etc. \\
Topography & Human body topography & String \\
Diagnostisc & Medical diagnostic. & Free text. \\
Advise & Medical advise & Free text \\
Language & A language of the metadata description. & ISO 3166: two letter \\
Description date & Image date description. & ISO 8601 \\
& & (yyyy-mm-dd) \\
\hline
\end{tabular}

\section{Author Class}

One or more authors may describe an image, and they can be people or institutions. The author class has properties that represents the author of the image. Person and Institution are sub-classes of Author. Properties of this class are name and e-mail.

\section{Relation Class}

The relation class represents a reference to a related resource. An image relation is: is related with, is format of, is part of and has part of. The relation can be among images, urls and another resources. 


\section{Annotations Class}

Annotation class represents components that can be highlighted and described in areas of the image. Attributes of the annotation class are: title, description, and X, Y coordinates.

\section{Medwords Class}

Medical terms and their values are always dependent of medical areas. The medwords class represents specific metadata not present in MedISeek model. The effective use of this class depends on the adoption of a dictionary of medical terms.

\subsection{A metadata model example}

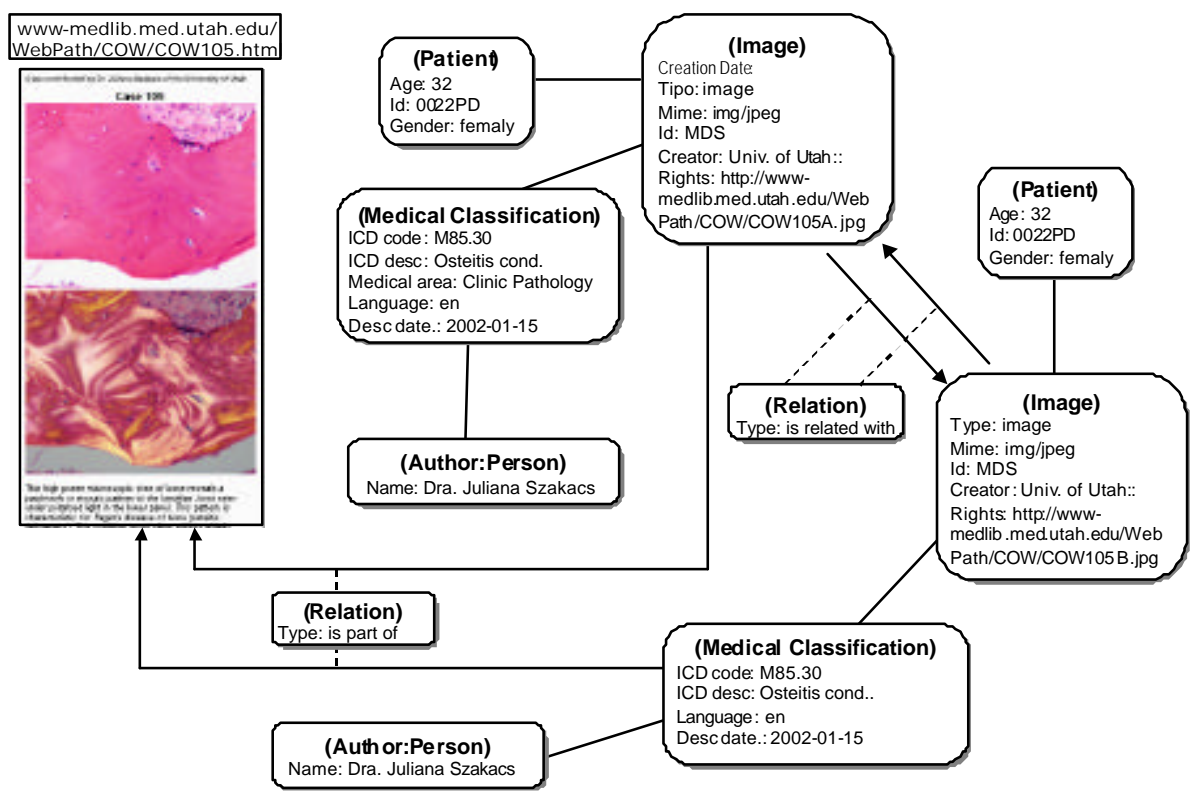

Figure 2. Instance diagram example

Figure 2 represents a diagram of an instance of the metadata model proposed. Two images, a web page and their relations are described utilizing the schema proposed in the MediSeek metadata model. 


\section{MedISeek Prototype}

The MedISeek prototype architecture is based on available web services (e.g. TCP/IP, etc.), allowing light coupling among sub-systems. The sub-systems interaction show the viability of the proposed metadata model, allowing to share and inter-operate resources on the web.

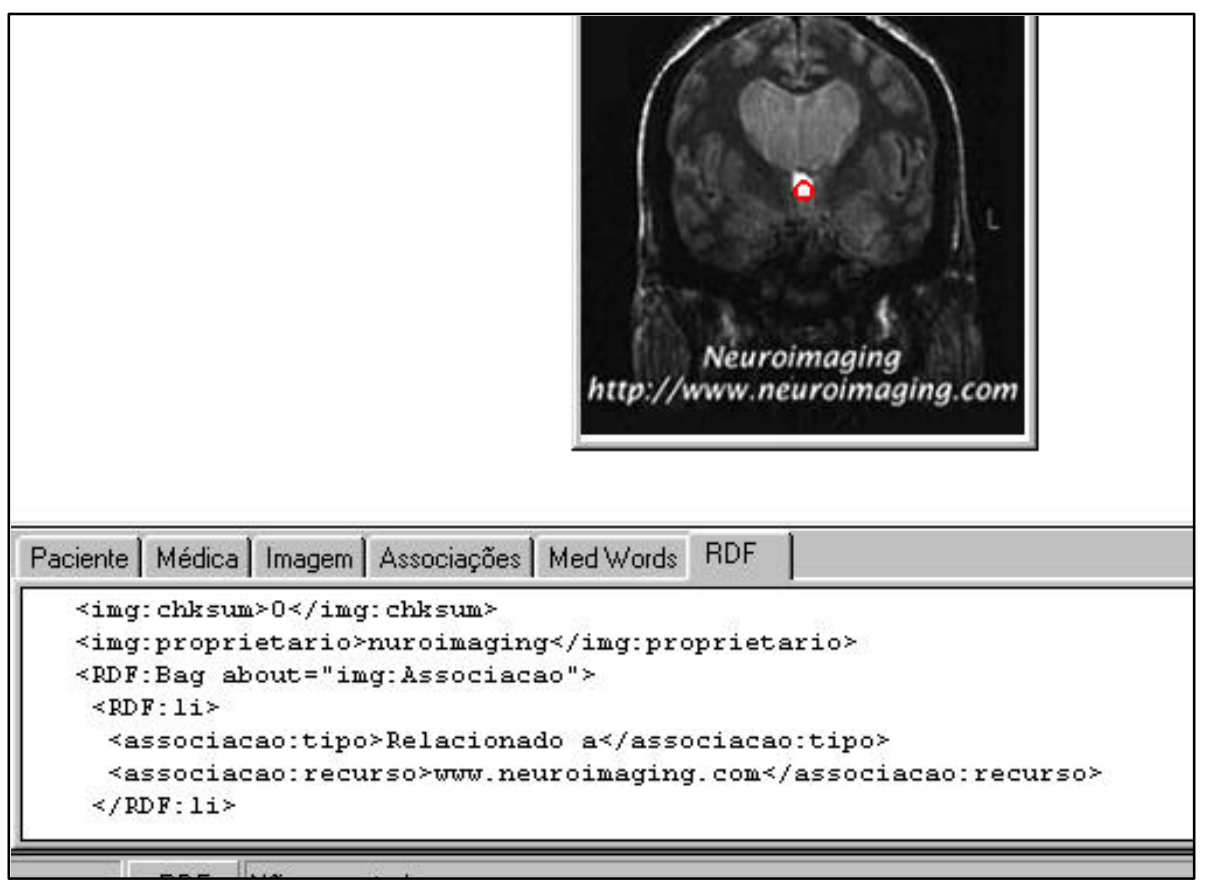

Figure 3. RDF fragment to be embedded in image header

Where to store the metadata was a decision we had to make when designing the prototype. Two solutions seemed viable: use of a relational database to assign metadata to the images, or embedding the metadata in header of the images. The first solution, although simple and very adopted in PACS systems, would not solve by itself the interoperability and image exchange problem (because images have been isolated from their descriptions). The adopted decision was a composition of the two solutions: embedding the metadata (converted in RDF) in the image headers (see Figure 3), and also store the metadata in a relational database to improve the search performance.

The use of a client-server architecture, inherent to the internet, solved the image sharing problem and presented as positive consequence decreasing of one of the largest problems of the image text annotation: high cost of manual description [13]. The MedISeek 
prototype operates based on voluntary contributions, therefore the images are described in a distributed fashion.

The following technologies were used in the prototype implementation: object oriented programming, RDF, XSL, sockets, dynamic pages in HTML, JPEG files architecture and relational database. The MedISeek architecture is composed by four subsystems: descriptor, manager, viewer and search module (Figure 4). The first sub-system offers support to image description based on the MedISeek metadata model. The image description is inserted in the image file header, and transmitted to the manager sub-system. The manager sub-system maintains a database with the image descriptions, and copies of the files for future retrieval, as well as for data persistency. Retrieval is performed by the search module and showed by the viewer sub-system in a web browser.

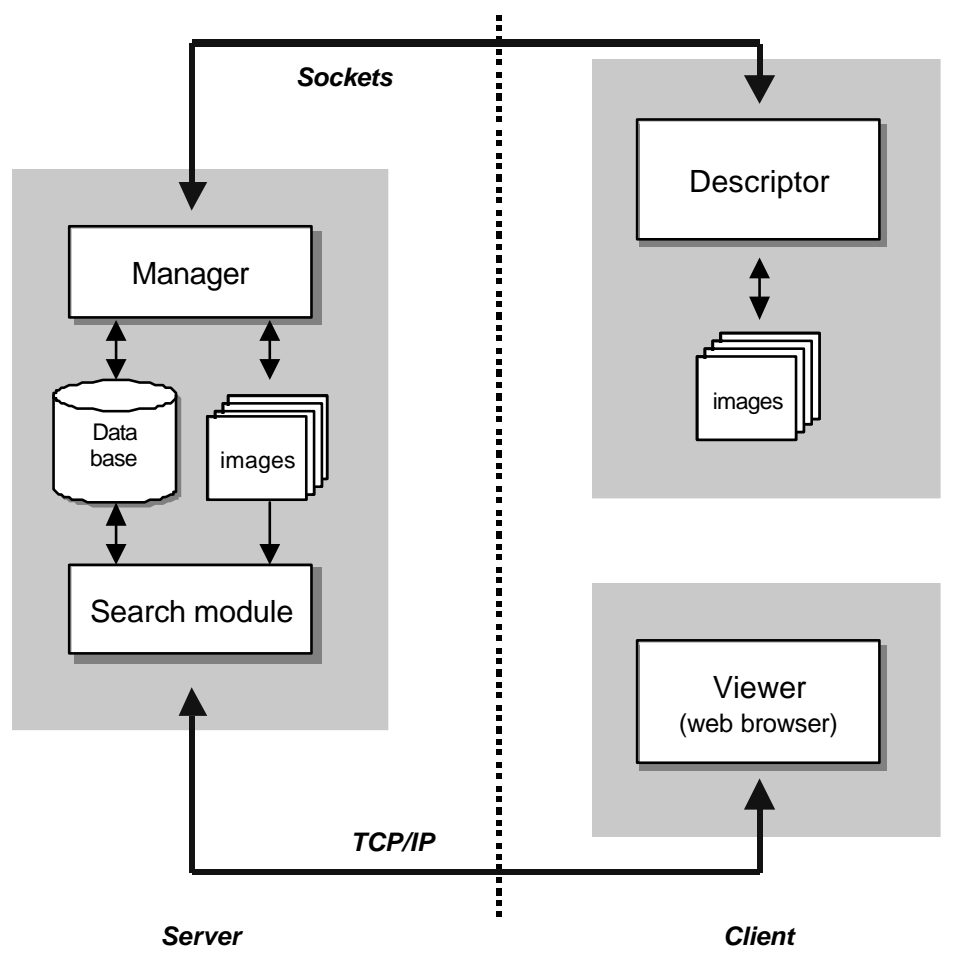

Figure 4 - MediSeek sub-systems diagram

A client-server architecture, with socket connections, is used to link the Descriptor and Manager sub-systems, implementing the image transport environment. The connection between the Search module and the Viewer sub-system is accomplished through web services. 
Next, the sub-systems of the MedISeek prototype are detailed.

\subsection{Descriptor sub-system}

Descriptor sub-system was developed in Borland $\mathrm{C}++$ Builder for Microsoft Windows, and offers the interface for the image description (Figure 5), as well as support for the connection with the manager sub-system. Basic functions of this sub-system are: images description interface; annotations module; RDF parser; ICD-10 interactive browser; RDF embedding and extraction module and connection module into manager sub-system

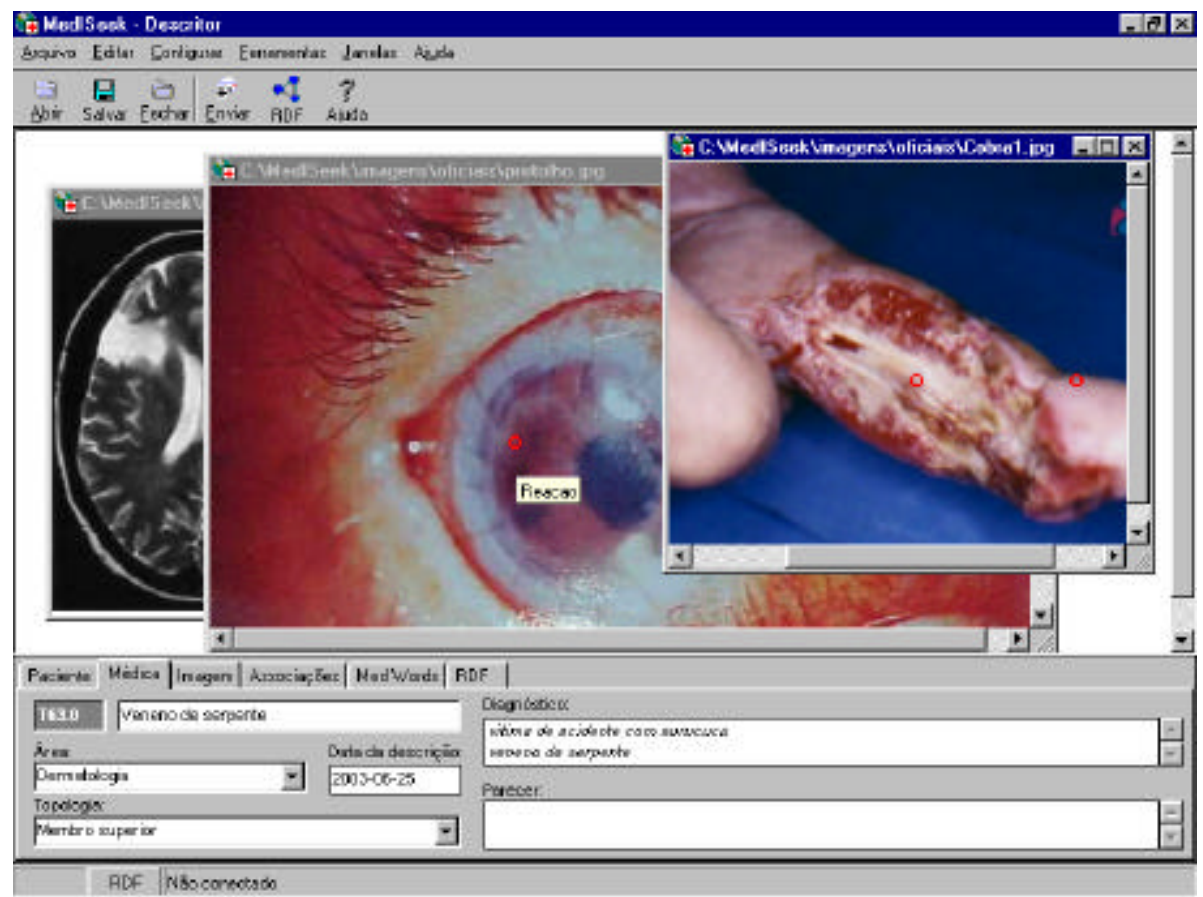

Figure 5. Descriptor sub-system user interface.

\subsection{Manager sub-system}

It was developed in $\mathrm{C}$ language for the Lynux platform. The manager sub-system main function is to extract RDF metadata from image's header, and convert them to relational database rows. This sub-system uses a MySQL database. A stream socket connection links this sub-system to the Descriptor sub-system, and the use of threads makes possible to serve multiple clients. 


\subsection{Search module}

Image retrieval is performed by searching through the Search module. Search module is a dynamic HTML application, developed using the PHP language and allows three types of searches: simple search (single attribute), ICD-10 search and detailed search (several attributes) (Figure 6). This module connects with the MySQL database, and implements queries with boolean expressions. Query results may be presented as thumbnails, allowing the image preview. A detailed image view is performed by the Viewer sub-system.

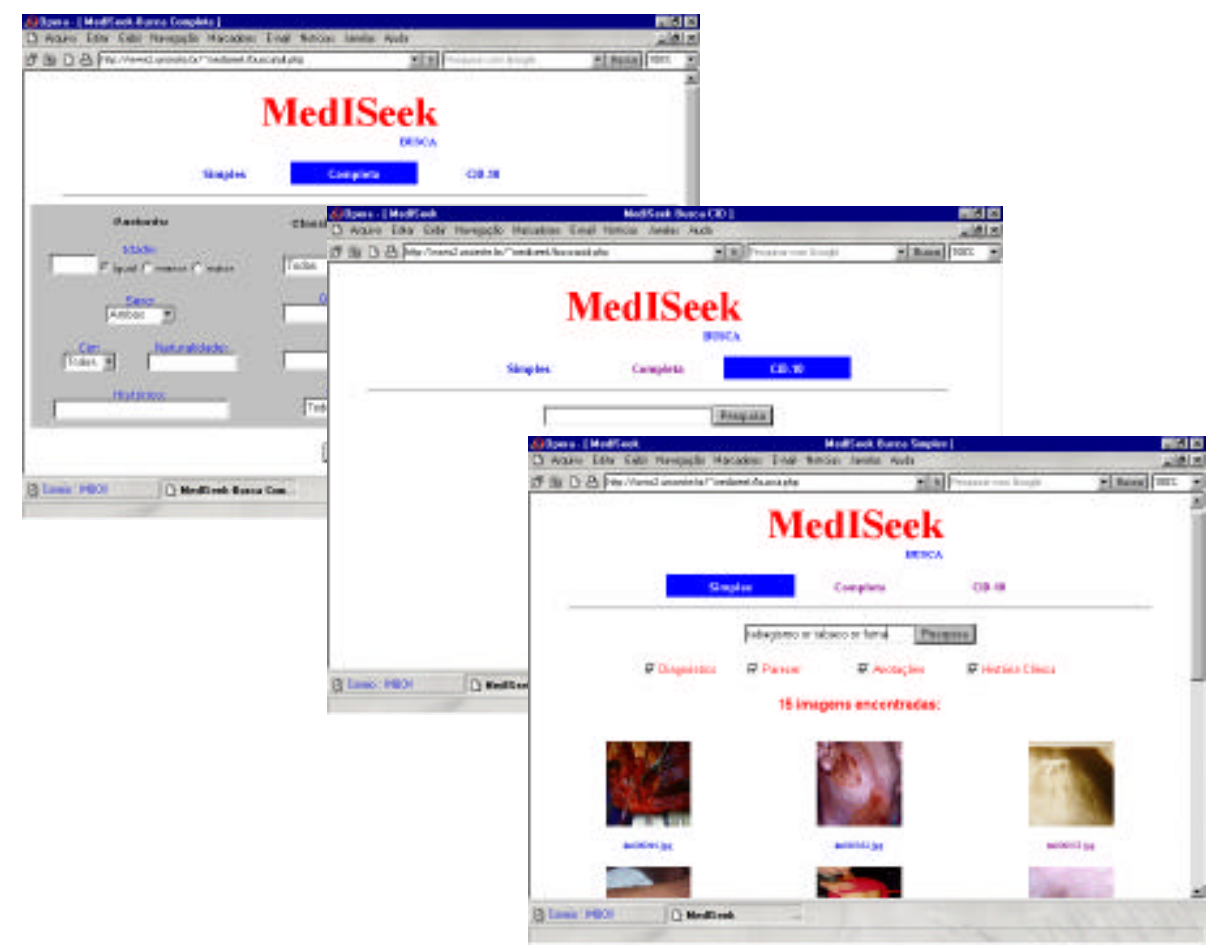

Figure 6 - Search pages.

\subsection{Viewer sub-system}


The Viewer sub-system details the selected images. The detailing process involves the RDF extraction from the image and its conversion into HTML. This process uses a XSL stylesheet. Figure 7 represents a Viewer sub-system interface.

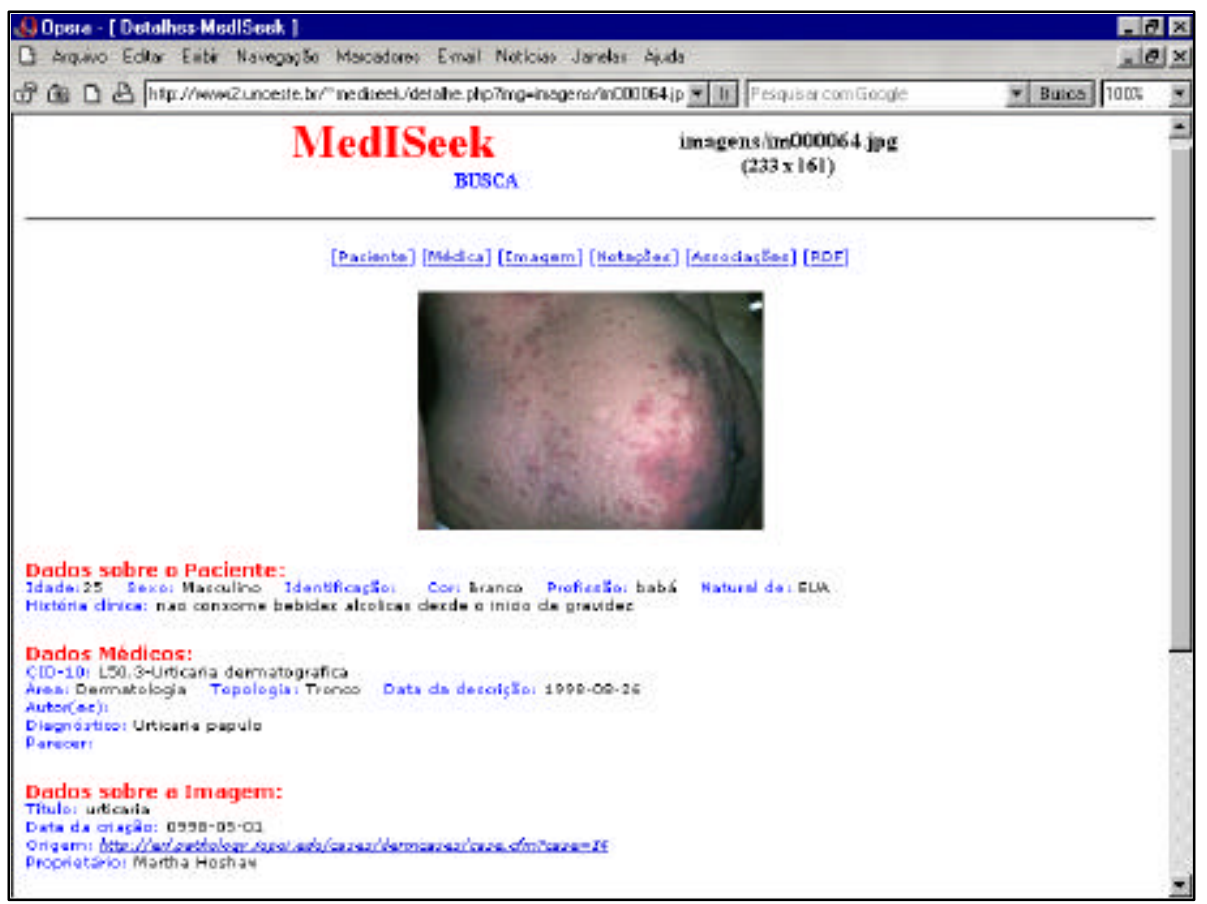

Figure 7 - Viewer sub-system interface

\section{$4 \quad$ Tests and Results}

Fifty images of clinical dermatology cases were inserted in MedISeek system to perform initial tests. A query example for images in dermatology, where the patient is male with tabagism antecedents, resulted in five retrieved images (Figure 8), and only one was not relevant (it was from a no smoker patient image). The search module generates the following SQL query: SELECT im_cod FROM imagem WHERE pa_sexo = "Male" AND (pa_histo LIKE "\%tobacco\%" OR pa_histo LIKE "\%tabagism\%" OR pa_histo LIKE "\%smoker\%" ) AND me_area = "Dermatology" ‘. 


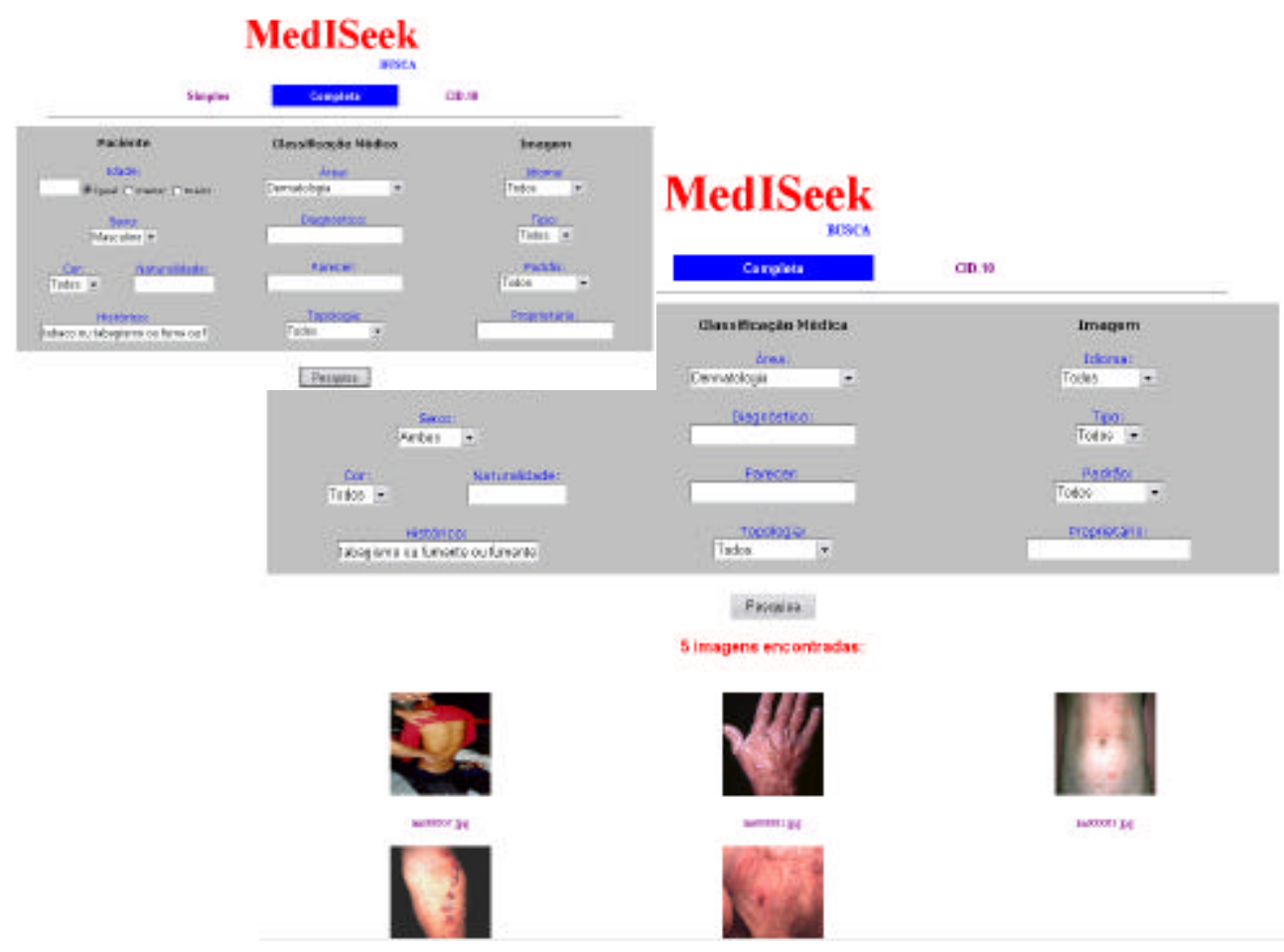

Figure 8 - Search and View process.

Later, the same query was performed on traditional web search systems, like Altavista, Google and Alltheweb (Figure 9), using the keywords: "'clinical case' and dermatology and man and (smoke or tobacco or tabagism)". Table 2 shows the results and demonstrates the superior precision of the system MedISeek for medical image retrieval in relation to other retrieval systems.

Table 4. Query results

\begin{tabular}{lcccc}
\hline \multicolumn{1}{c}{ System } & Results & Important results & Precision results & Precision (\%) \\
\hline MedISeek & 5 & 5 & 4 & $80 \%$ \\
Altavista & 24 & 2 & 1 & $4,1 \%$ \\
Google & 64 & 1 & 1 & $1,5 \%$ \\
Altheweb & 34 & 0 & 0 & $0 \%$ \\
\hline
\end{tabular}



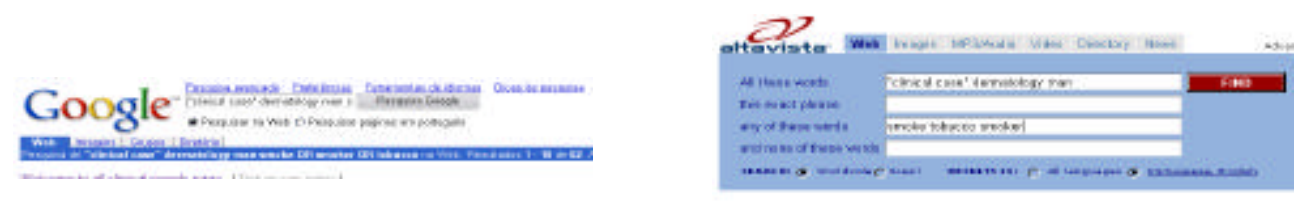

\section{S110 110 advanced search $::$ customize preferences $::$ submit site $::$ help 000 find it all 00 . edit wour advanced search \begin{tabular}{|l|l|l|l|}
\hline Web & News & Pictures | Video | Audio | FTP files
\end{tabular}}

Figure 9 - Medical image retrieval in traditional web search engines

Other tests were performed comparing the MedISeek system with other systems in specific medical areas. The John Hopkins University, in Baldimore, maintains a web image database named "Dermatlas.org" (http://dermatlas.med.jhmi.edu/derm). Actually, the system contains 4610 dermatology images. The performances of MedISeek and Dermatlas were compared. The query was a search for images of HIV positive males. The results are displayed in table 5 .

Table 5. Query results

\begin{tabular}{lcccc}
\hline \multicolumn{1}{c}{ System } & Results & Not repeated results & Precision results & Precision $(\%)$ \\
\hline MedISeek & 14 & 13 & 11 & $79 \%$ \\
DermAtlas & 32 & 13 & 7 & $22 \%$ \\
\hline
\end{tabular}

Several images recovered by the DermAtlas system reported the same clinical case, but this system could not differentiate some keywords, such as HIV and HIVES, or man and woman. Only three images were discarded as irrelevant from the images retrieved by the MediSeek system: one duplicate image (same clinical case), a HIV negative patient and a clinical case of a children with HIV positive parents.

Tests with a larger group of images will be reported elsewhere, and in several medical areas. The retrieval image precision in the MedISeek system depends mainly on the precision of the image description, mainly in the free text attributes. Use of a thesaurus dictionary to aid free text attributes description should help improving precision and retrieval.

\section{Concluding Remarks}

This work presents a metadata model for sharing medical image resources through the web, and shows a prototype based on this model for image retrieval and visualization. This 
metadata model is compatible with the ICD-10, which is fundamental for the relevant facts (i.e. diseases) description precision and clinical investigation data/knowledge distribution.

The main motivation for this metadata model and the MedISeek prototype was to design a solution that provides access to relevant cases documented by medical images and their associated data, which are often confined to resources available within some medical institutions. Also, the RDF use and the image embedded metadata allows the generated resources to be exchanged with other systems. The existent independence between the metadata model and the image format makes it easier to extend its uses to others image format. Also, a cooperative and distributed environment can be constituted through the process of annotations and sharing of images. Actually, currently the MedISeek prototype uses only the JPEG format for images. DICOM format actualy is not suported, but is possible to compatibilize the MedISeek data model and DICOM data model throw the correlation among their metadata.

Compared to used methods of search on the web, such google and altavista, the proposed ontology provides a very superior precision when used to indexing and searching medical images.

In the near future we intend to test the MedISeek system in a network of University Hospitals, linked to Brazilian Federal Universities, enabling the creation of a new tool for distance learning and medical information exchange. This may be particularly useful for fast medical knowledge/information distribution in wide areas.

\section{References}

[1] National Center for Health Statistics. http://www.cdc.gov/nchs/about/major/dvs/icd10des.htm

[2] Bozec, C.L. et al. IDEM: a Web application of case-based reasoning in histopathology. Computers in Biology and Medicine, May 1998, 473-487.

[3] History of Medical Diagnosis and Diagnostic Imaging. http://imaginis.com/faq/history.asp

[4] Dublin Core Metadata Initiative. http://dublincore.org

[5] MARC Standarts: Library of Congress Network Development and MARC Standard Office. http://www.loc.gov/marc/

[6] SAIF \& FMEEBC. http://home.gdbc.gov.bc.ca/fmebc/

[7] Text Encoding Initiative. http://www.tei-c.org/

[8] The Summary Object Interchange Format (SOIF), http://harvest.sourceforge.net/harvest1.4.pl2-docs/node151.html

[9] Meta Content Framework (MCF), http://www.ukoln.ac.uk/metadata/ resources/mcf/ 
[10] Lagoze, Carl. The Warwick Framework: A Container Architecture for Diverse Sets of Metadata, D-Lib Magazine, July/August 1996.

[11] Resource Description Framework (RDF), http://www.w3.org/RDF/

[12] Zanete, Nelson Henrique. Uma Avaliação de Abordagens Alternativas para Armazernar RDF em Banco de Dados Relacional. MSc Thesis, PPGC, UFRGS, Porto Alegre, Brazil, 2001, 91p.

[13] Chang et al. Multimedia access and retrieval (panel session): the state of the art and future directions. Proceedings of the seventh ACM International conference on Multimedia October 30 - November 5, 1999, Orlando, FL USA, pp 443 - 445. 